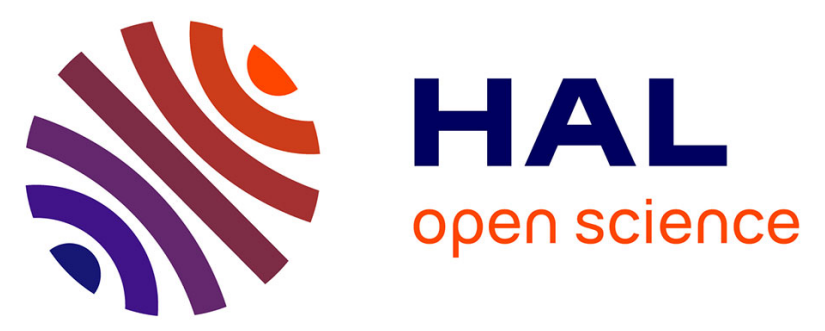

\title{
Buffer free InGaAs quantum well and in-plane nanostructures on InP grown by atomic hydrogen assisted MBE
}

A. Bucamp, Christophe Coinon, J.-L. Codron, David Troadec, X. Wallart, L. Desplanque

\section{To cite this version:}

A. Bucamp, Christophe Coinon, J.-L. Codron, David Troadec, X. Wallart, et al.. Buffer free InGaAs quantum well and in-plane nanostructures on InP grown by atomic hydrogen assisted MBE. Journal of Crystal Growth, 2019, 512, pp.11-15. 10.1016/j.jcrysgro.2019.01.033 hal-02391386

\section{HAL Id: hal-02391386 \\ https://hal.science/hal-02391386}

Submitted on 21 Oct 2021

HAL is a multi-disciplinary open access archive for the deposit and dissemination of scientific research documents, whether they are published or not. The documents may come from teaching and research institutions in France or abroad, or from public or private research centers.
L'archive ouverte pluridisciplinaire HAL, est destinée au dépôt et à la diffusion de documents scientifiques de niveau recherche, publiés ou non, émanant des établissements d'enseignement et de recherche français ou étrangers, des laboratoires publics ou privés.

\section{(ㅇ)(1) $\$$}

Distributed under a Creative Commons Attribution - NonCommerciall 4.0 International 


\title{
Buffer free InGaAs quantum well and in-plane nanostructures on InP grown by atomic hydrogen assisted $\mathrm{MBE}$
}

\author{
A.Bucamp, C.Coinon, J.-L.Codron, D.Troadec, X.Wallart and L.Desplanque* \\ Univ. Lille, CNRS, Centrale Lille, ISEN, Univ. Valenciennes, UMR 8520 - IEMN, F-59000 \\ Lille, France \\ *ludovic.desplanque@univ-lille.fr
}

\begin{abstract}
We investigate the optical and electrical properties of InGaAs thin films and nanostructures grown directly on an InP semi-insulating substrate without any buffer layer using atomic hydrogen assisted molecular beam epitaxy. We confirm the positive influence of the atomic hydrogen flux during the deoxidization process as well as during the growth itself improving the photoluminescence properties of InGaAs quantum wells. We also study the effect of the atomic hydrogen flux on the electrical properties of buffer free undoped, Te or Si doped InGaAs epilayers. Eventually, we demonstrate that atomic hydrogen flux can be used to achieve InGaAs growth selectivity with respect to a $\mathrm{SiO}_{2}$ mask for a growth temperature as low as $470^{\circ} \mathrm{C}$ and obtain in-plane InGaAs nanostructures on $\mathrm{InP}$ with good transport properties.
\end{abstract}

KEYWORDS: A3.Selective epitaxy; A1.Nanostructures; B2.Semiconducting indium compounds; A3.Molecular beam epitaxy; A3.Quantum wells

\section{Introduction}

Whereas metal organic vapor phase epitaxy (MOVPE) has demonstrated its efficiency for selective area growth (SAG) of InGaAs nanostructures lattice matched to InP with respect to a 
dielectric mask [1] [2], very few results has been reported using molecular beam epitaxy (MBE). This is mainly due to the difficulty to gather the growth conditions required to achieve both Gallium re-evaporation from the hard mask and low Indium re-evaporation in the InGaAs layer. For a rather low growth rate $(0.04 \mu \mathrm{m} / \mathrm{h})$, a temperature as large as $600^{\circ} \mathrm{C}$ is for instance needed to reach a good selectivity for GaAs growth with respect to a $\mathrm{SiO}_{2}$ mask [3], whereas InGaAs is known to experience a strong re-evaporation of In and As at such a high growth temperature. Migration enhanced epitaxy can be used to achieve selectivity with respect to a $\mathrm{SiO}_{2}$ mask for $\mathrm{InGaAs}$ lattice matched to $\mathrm{InP}$ at $560^{\circ} \mathrm{C}$ [4]. It has also been shown that an atomic hydrogen flux can help (i) to reach selective growth of $\operatorname{In}_{0.53} \mathrm{GaAs}_{0.47} / \mathrm{InP}$ layers at $560^{\circ} \mathrm{C}$ [5] and the one of $\mathrm{GaAs}$ pyramidal structures at $630^{\circ} \mathrm{C}$ supporting $\operatorname{In}_{0.2} \mathrm{Ga}_{0.8} \mathrm{As}$ quantum dots grown at $520^{\circ} \mathrm{C}$, thanks to a $\mathrm{SiO}_{2}$ micro-patterning of the substrate, [6]. More

recently, we have demonstrated that an atomic hydrogen flux strongly improves the selectivity for $\mathrm{GaSb}$ or $\mathrm{InSb}$ at a growth temperature as low as $470^{\circ} \mathrm{C}$ and $400^{\circ} \mathrm{C}$ respectively [7] [8] [9]. In this paper, we show that good selectivity with respect to a $\mathrm{SiO}_{2}$ mask can be obtained at $470^{\circ} \mathrm{C}$ for InGaAs grown on an $\mathrm{InP}(001)$ substrate using atomic hydrogen assisted $\mathrm{MBE}$. The photoluminescence (PL) of InGaAs/InP and InGaAs/InAlAs quantum wells (QW) as well as Hall, transmission line method (TLM) and four probe measurements are carried out to determine the impact of the atomic hydrogen flux on the optical and electrical properties of InGaAs two dimensional layers and in-plane nanostructures.

\section{Experimental}

Epi-layers were grown using two MBE systems: a RIBER 32P, equipped with Arsine and Phosphine High Temperature Injectors for $\mathrm{As}_{2}$ and $\mathrm{P}_{2}$ sources, is used for $\mathrm{InGaAs} / \mathrm{InP}$ heterostructures without atomic hydrogen flux, whereas a RIBER Compact21 equipped with an $\mathrm{As}_{4}$ solid source and a RF plasma cell, is used for atomic Hydrogen assisted epitaxy. These 
two systems are connected with each other under ultra-high vacuum so that one epi-layer can be grown using the two systems.

The impact of the atomic hydrogen flux on the deoxidization of InP substrate was first investigated by the growth of an InGaAs/InP QW layer with four different surface preparations prior to the growth (figure 1a). For the first sample (sample A), substrate deoxidization has been performed by heating the surface up to $520^{\circ} \mathrm{C}$ under a $\mathrm{P}_{2}$ flux and a $300 \mathrm{~nm}$ thick $\mathrm{InP}$ buffer layer has been grown at $500^{\circ} \mathrm{C}$ followed by $30 \mathrm{~nm} \mathrm{In}_{0.53} \mathrm{Ga}_{0.47} \mathrm{As}$ and a $70 \mathrm{~nm}$ InP top barrier. For sample B, the same method has been used for deoxidization but no buffer has been grown before InGaAs and top InP layer (with the same thicknesses than for sample A). Sample C has the same structure than sample B but with a deoxidization under $\mathrm{As}_{4}$ flux at $520^{\circ} \mathrm{C}$. Eventually, for sample D, we used a deoxidization under $\mathrm{As}_{4}$ and atomic hydrogen flux at $480^{\circ} \mathrm{C}$ for the surface preparation and no buffer layer prior to InGaAs/InP layer as for samples B and C.

In a second set of samples ( $\mathrm{E}$ and $\mathrm{F}$ ), we investigated the impact of atomic $\mathrm{H}$ on the properties of an InGaAs/InAlAs QW. This QW is composed of a $30 \mathrm{~nm}$ thick $\mathrm{In}_{0.53} \mathrm{Ga}_{0.47} \mathrm{As}$ layer sandwiched between a $300 \mathrm{~nm} \operatorname{In}_{0.52} \mathrm{Al}_{0.58} \mathrm{As}$ buffer layer and a $70 \mathrm{~nm} \operatorname{In}_{0.52} \mathrm{Al}_{0.58} \mathrm{As}$ top barrier. To avoid oxidization of $\mathrm{Al}$ containing layers, a top $5 \mathrm{~nm} \operatorname{In}_{0.53} \mathrm{Ga}_{0.47} \mathrm{As}$ cap was used. For samples A to F, a growth rate of 1 ML.s ${ }^{-1}$ was used.

The transport properties of $\operatorname{In}_{0.53} \mathrm{Ga}_{0.47} \mathrm{As}$ layers on InP SI substrate with different thicknesses and doping were then investigated. Undoped and n-doped layers using Si or Te dopants were studied. Table 1 summarizes the parameters used for the growth of this G-series of samples. Their transport properties were analyzed with Hall measurements using Van der Pauw mesa that were formed either with a top-down approach by etching a 2D layer or with a bottom-up method by growing directly the desired shape of the mesa by atomic hydrogen assisted selective area growth. The typical dimensions of the Van de Pauw mesa is $1.25 \mathrm{mmx} 1.25 \mathrm{~mm}$ 
for etched samples and $9 \mu \mathrm{m} \times 9 \mu \mathrm{m}$ for selective area grown samples. Details about the $\mathrm{SiO}_{2}$ mask preparation can be found in reference [10]. The nominal growth rate was reduced to 0.2 ML.s ${ }^{-1}$ to ensure a good selectivity with respect to the mask.

To analyze the transport properties inside selective area grown in-plane InGaAs nanostructures, some of these SAG samples (G10 and G11) exhibits also patterns consisting of $100 \mathrm{~nm}$ wide stripes with different lengths (from $3 \mu \mathrm{m}$ to $12 \mu \mathrm{m}$ ) to perform TLM measurements and extract resistivity of SAG nanowires. $100 \mathrm{~nm}$ wide connected stripes were also designed to perform four probe measurements and deduce the resistivity of undoped nanostructures independently from the large contact resistance. For these devices, electron beam lithography was used to deposit Ti/Au contacts with a constant $1 \mu$ m overlap with the nanostructure.

\section{Optical characterization of InGaAs QW}

Figure $1 \mathrm{~b}$ displays the room temperature (RT) PL spectra measured the InGaAs/InP QWs with different deoxidization process (sample A to D). Of course, the reference sample A grown with standard deoxidization under $\mathrm{P}_{2}$ flux and with a $300 \mathrm{~nm} \mathrm{InP}$ buffer layer exhibits the largest PL intensity. When InGaAs QW is grown directly on the thermally deoxidized surface under $\mathrm{P}_{2}$ without any buffer layer, the PL intensity is strongly reduced by almost 3 orders of magnitude (sample B). This degradation is even more important when the thermal deoxidization is performed under $\mathrm{As}_{4}$ flux (sample C). However, when atomic hydrogen is used during the deoxidization of the InP substrate at $480^{\circ} \mathrm{C}$ and under an $\mathrm{As}_{4}$ flux, the PL intensity is only divided by a factor 4 with respect to the reference sample despite the absence of buffer layer. This positive impact can be attributed to a reduction of the surface roughness induced by annealing and the carbon contamination from the substrate surface using low temperature atomic hydrogen assisted deoxidization [11] [12]. With this deoxidization 
process, high quality material can thus be contemplated when grown directly on the substrate surface without any buffer layer.

For these samples, the atomic hydrogen flux was not present during InGaAs growth. Its impact on the optical properties of InGaAs was investigated using sample E and F. As can be

seen in figure 2, the presence of an atomic hydrogen flux during the growth does not induce any degradation of the PL intensity, a slight improvement is even observed.

\section{Electrical characterization}

The transport properties measured by Hall effect on G samples are summarized in table 1 and plotted in figure 3. On the whole, these data follow a standard degradation of the electron mobility when the doping is increased. It is interesting to notice that the value measured on Te or Si doped samples grown with an atomic hydrogen flux or not, or with a top-down or a bottom-up fabrication exhibits the same evolution. This tendency is also very consistent with the undoped samples grown under an atomic hydrogen flux which exhibits a Hall mobility of $10500 \mathrm{~cm}^{2} \cdot \mathrm{V}^{-1} \cdot \mathrm{s}^{-1}$ at RT (and $25400 \mathrm{~cm}^{2} \cdot \mathrm{V}^{-1} \cdot \mathrm{s}^{-1}$ at $77 \mathrm{~K}$ ) for a constant Hall density of $3.5 \times 10^{15} \mathrm{~cm}^{-3}$. The Hall mobility measured on the undoped InGaAs layer grown without any atomic $\mathrm{H}$ flux is much lower with $4650 \mathrm{~cm}^{2} \cdot \mathrm{V}^{-1} \cdot \mathrm{s}^{-1}$ at $\mathrm{RT}$ and $2720 \mathrm{~cm}^{2} \cdot \mathrm{V}^{-1} \cdot \mathrm{s}^{-1}$ at $77 \mathrm{~K}$, the Hall density experiencing a small decrease from $-2 \times 10^{15} \mathrm{~cm}^{-3}$ to $-1.1 \times 10^{15} \mathrm{~cm}^{-3}$ respectively. This quite unexpected result may come from a small carbon contamination of the InGaAs layer causing a low p-type doping. The combination of hole and electron transport inside the layer thus lead to a lower Hall mobility and density at RT. At $77 \mathrm{~K}$, the intrinsic electron density is reduced, resulting in a larger influence of hole transport properties and thus in a decrease of the Hall mobility. This interpretation and the results obtained on G02 sample mean that the use of an atomic hydrogen flux probably reduce p-type contamination (residual carbon concentration on the surface after deoxidization) during the growth. 
Let's now turn to the selective area grown nanostructures. Figure 4 shows some plan view scanning electron micrographs of different InGaAs nanostructures observed on samples G10 and G11. Figure 4a and $4 \mathrm{~b}$ corresponds to the nanostructures obtained after the growth inside $100 \mathrm{~nm}$ wide apertures inside the $\mathrm{SiO}_{2}$ mask. As can be seen, a very good selectivity is achieved and well-defined facets are formed all along the nanostructure. Cross-section Scanning Transmission Electron Micrographs at $30 \mathrm{keV}$ (figure 4c and 4d) performed after thin slices preparation using Focus Ion Beam (FIB) show that nanostructures grown along the [110] crystallographic orientation exhibit $\{111\}_{\mathrm{B}}$ side facets and $\{001\}$ top surface. In the perpendicular orientation, $\{113\}_{\mathrm{A}}$ and small $\{111\}_{\mathrm{A}}$ facets are observed. This is quite similar than what we have already observed for InAs selective area homoepitaxy [10]. The crosssection area of these nanostructures is approximately 5670 and $5620 \mathrm{~nm}^{2}$ for [110] and [1-10] oriented wires respectively. This is much larger than the expected value for the deposition of a nominal thickness of $30 \mathrm{~nm}$ inside a $100 \mathrm{~nm}$ wide aperture $\left(3000 \mathrm{~nm}^{2}\right)$ for two reasons: (i) the HF treatment used to fully opened the $100 \mathrm{~nm}$ mask aperture has enlarged the width to more than $140 \mathrm{~nm}$ and (ii) the $3 \mathrm{D}$ shape of the nanostructures results in a larger surface collecting the molecular beams and thus increases the growth rate.

Figures 4e-h display the morphology of selective area grown patterns used for four probe and Van der Pauw measurements (Fig. 4g). As illustrated in figure 4h, four of the six branches of a $100 \mathrm{~nm}$ wide nanostructure are used to inject current in the in-plane nanowire and measure the resulting voltage between each extremity of the central arm.

The resistances measured for individual NW as shown in figure $4 \mathrm{a}$ and $4 \mathrm{~b}$ with different interelectrode spacing are plotted in figure 5 for the two crystallographic orientations. From this TLM measurement, one can estimate the values of the contact resistances and the resistance per unit length of the in-plane NW. For the [110] oriented NW, a contact resistance of $375 \Omega$ and a linear resistance of $880 \Omega . \mu \mathrm{m}^{-1}$ are deduced. For the [1-10] oriented NW, these values 
are $125 \Omega$ and $570 \Omega . \mu \mathrm{m}^{-1}$ respectively. Assuming the cross-section areas estimated from figure $4 \mathrm{c}$ and $4 \mathrm{~d}$, this corresponds to a bulk conductivity of 2022 and $3112 \mathrm{~S} . \mathrm{cm}^{-1}$ respectively. This is significantly lower than the Hall conductivity measured on large Van der Pauw device $\left(3608{\mathrm{~S} . \mathrm{cm}^{-1}}^{-1}\right.$. The 3D faceting of the nanostructures could explain this result as the migration of III-element adatoms from different facets results in a larger effective growth rate than the nominal one, and the different dangling bonds of each facet may result in different way and efficiency for Si incorporation inside the nanostructure [10].

For the undoped sample (G11), such a TLM measurement was not possible as the resistance of the nanowires was dominated by the contact one (larger than $500 \mathrm{k} \Omega$ ). We then used the nanostructured presented on figure $4 \mathrm{e}$ and $4 \mathrm{f}$ and the measurement set-up of figure $4 \mathrm{~h}$ to measure the resistance of the NW independently from the contacts. Quite similar conductivities of 24.4 and $26.3{\mathrm{~S} . \mathrm{cm}^{-1}}^{-}$were deduced for a $1 \mu \mathrm{m}$ long central arm oriented along [110] and [1-10] directions respectively. This is quite expected as no doping incorporation is involved in the growth process for this sample. This value of conductivity is reported on the previous graph summarizing the Hall measurements of table 1 (figure 6). If we assume the evolution of electron mobility with respect to the electron density measured on

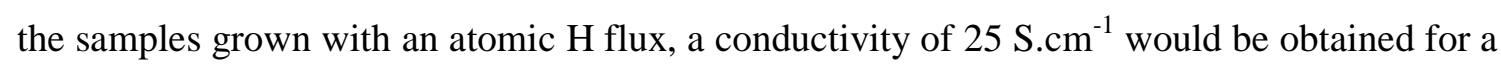
mobility of $8800 \mathrm{~cm}^{2} \cdot \mathrm{V}^{-1} \cdot \mathrm{s}^{-1}$ and an electron density of $1.7 \times 10^{16} \mathrm{~cm}^{-3}$.

\section{Conclusion}

The optical and electrical properties of InGaAs grown by atomic hydrogen assisted MBE have been investigated. We have confirmed the positive impact of an atomic Hydrogen flux both on the deoxidization of the InP surface as well as during the growth as it improves the PL intensity of InGaAs/InAlAs QW and the Hall mobility in undoped InGaAs bulk layers whereas it does not affect transport properties of Te or Si doped epilayers. Furthermore, the 
atomic hydrogen flux allows the selective area $\mathrm{MBE}$ of InGaAs nanostructures on InP substrate at $470^{\circ} \mathrm{C}$, with an estimated RT bulk electron mobility of $8800 \mathrm{~cm}^{2} . \mathrm{V}^{-1} \cdot \mathrm{s}^{-1}$ inside inplane undoped NW whereas Si-doped ones experience a lower silicon incorporation due to 3D faceting. We believe that this growth mode could be very useful for the fabrication of in-plane nanostructures for low electron effective mass transistors or mesoscopic nanodevices.

\section{Acknowledgments}

The authors would like to acknowledge financial support from the national research agency under the DIRAC-III-V project (contract No.: ANR-16-CE24-0007), the French Technological Network Renatech, and the Région Hauts de France.

References

[1] N. Waldron et al., Solid-State Electronics 115, pp. 81-91, 2016.

[2] C.Zota et al., ACS Nano 9, p. 9892-9897, 2015.

[3] A.Okamoto et al., Journal of Applied Phys. 66, p. 3413, 1989.

[4] M.A.Wistey et al., J. of Vacuum Science \& Tech. B 33, p. 011208, 2015.

[5] Kuroda et al.,Indium Phosphide and Related Materials, 1993. Conference Proceedings., Fifth International Conference on, pp. 52-55, 1993.

[6] Y. Suzuki et al, Jpn. J. Appl. Phys. 36 (1997) L1538.

[7] M.Fahed et al., Nanotechnology 27, p. 505301, 2016.

[8] M.Fahed et al., J.Cryst. Growth 477, p. 45, 2017.

[9] L.Desplanque et al., Nanotechnology 29, p. 305705, 2018.

[10] L.Desplanque et al., Nanotechnology 25, p. 465302, 2014.

[11] T. Sugaya and M. Kawabe, Jp. J. Appl. Phys. 30 (1991) L402.

[12] Y. Chuna, T. Sugaya, Y. Okada and M. Kawabe, Jpn. J. Appl. Phys. 32 (1993) L287. 


\section{Table caption:}

\section{Table 1.}

Parameters used for the growth and the Hall device fabrication of the G-series of samples dedicated to the transport measurements.

\section{Figure captions:}

\section{Figure 1.}

Description of the deoxidization process and epitaxial structure of the samples A, B , C and D (a) and corresponding room temperature PL spectra (b). The vertical dash line indicates the cut-off from the detector.

\section{Figure 2.}

PL spectra measured at room temperature on sample E (blue line) and F (red line).

\section{Figure 3.}

Hall mobility and density measured on the G-series of samples. Black symbols corresponds to the samples grown without atomic hydrogen flux. Red symbols corresponds to the samples 
grown under an atomic hydrogen flux and with a top-down Hall mesa fabrication. Blue symbols stand for the samples grown by atomic hydrogen assisted selective area epitaxy.

\section{Figure 4.}

Top view SEM images of InGaAs nanostructures grown by atomic hydrogen selective area epitaxy (sample G11) inside $100 \mathrm{~nm}$ wide $\mathrm{SiO}_{2}$ apertures oriented in the [110] (a) or [1-10] (b) directions. FIB-STEM cross-section images of the nanostructures shown in figure 4a (c) and $4 \mathrm{~b}(\mathrm{~d})$. Plan view SEM images of $100 \mathrm{~nm}$ wide InGaAs branched nanostructures used for four probe measurements (e and f). InGaAs Van der Pauw pattern grown using atomic hydrogen assisted selective area epitaxy (g). Scheme of the set-up used for measuring the conductivity of the central arm of a nanostructure such as the one shown in figure $4 \mathrm{e}$.

\section{Figure 5.}

TLM measurements on single $100 \mathrm{~nm}$ wide in-plane NW of sample G10 oriented in the [110] (square) or [1-10] (down triangle) orientation. Red and blue lines are the respective linear regressions.

\section{Figure 6.}

Hall transport properties of the G-series samples compared to the conductivity (around 25 S.cm ${ }^{-1}$ ) deduced from four probe measurements on sample G11. The black dashed line is a guide for the eyes following the properties of atomic hydrogen assisted samples. The crossing

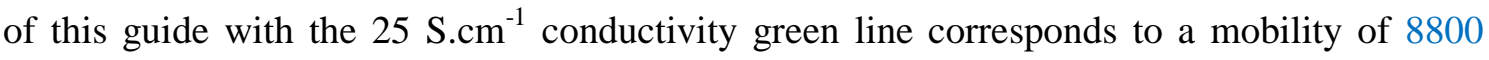
$\mathrm{cm}^{2} \cdot \mathrm{V}^{-1} \cdot \mathrm{s}^{-1}$ and a density of $1.7 \times 10^{16} \mathrm{~cm}^{-3}$. 
Table 1

\begin{tabular}{|c|c|c|c|c|c|c|}
\hline Sample & $\begin{array}{l}\text { Thickness } \\
(\mathrm{nm})\end{array}$ & $\begin{array}{l}\text { Atomic } \\
\text { Hydrogen }\end{array}$ & $\begin{array}{l}\text { Type of } \\
\text { doping }\end{array}$ & $\begin{array}{l}\text { Hall bar } \\
\text { fabrication }\end{array}$ & $\mu_{\text {Hall }}\left(\mathrm{cm}^{2} \cdot \mathrm{V}^{-1} \cdot \mathrm{s}^{-1}\right)$ & $\mathrm{n}_{\text {Hall }}\left(\mathrm{cm}^{-3}\right)$ \\
\hline G01 & 1000 & No & undoped & Top-down & $\begin{array}{c}4650 \text { (300K) } \\
2720 \text { (77K) }\end{array}$ & $\begin{array}{l}2 \times 10^{15}(300 \mathrm{~K}) \\
1,1 \times 10^{15}(77 \mathrm{~K})\end{array}$ \\
\hline G02 & 1000 & Yes & undoped & Top-down & $\begin{array}{c}10500 \text { (300K) } \\
25400 \text { (77K) }\end{array}$ & $\begin{array}{c}3.6 \times 10^{15}(300 \mathrm{~K}) \\
3,2 \times 10^{15}(77 \mathrm{~K})\end{array}$ \\
\hline G03 & 200 & No & $\mathrm{Si}$ & Top-down & 3470 & $4.1 \times 10^{18}$ \\
\hline G04 & 200 & Yes & $\mathrm{Si}$ & Top-down & 3520 & $4.25 \times 10^{18}$ \\
\hline G05 & 100 & Yes & $\mathrm{Si}$ & Top-down & 5610 & $4.0 \times 10^{17}$ \\
\hline G06 & 100 & Yes & $\mathrm{Te}$ & Top-down & 3560 & $3.3 \times 10^{18}$ \\
\hline G07 & 100 & Yes & $\mathrm{Te}$ & Top-down & 5940 & $2.5 \times 10^{17}$ \\
\hline G08 & 100 & Yes & $\mathrm{Te}$ & Bottom-up & 6450 & $1.6 \times 10^{17}$ \\
\hline G09 & 100 & Yes & $\mathrm{Si}$ & Bottom-up & 2840 & $7.5 \times 10^{18}$ \\
\hline G10 & 30 & Yes & $\mathrm{Si}$ & Bottom-up & 1280 & $1.6 \times 10^{19}$ \\
\hline G11 & 30 & Yes & undoped & Bottom-up & Not measurable & Not measurable \\
\hline
\end{tabular}




\section{Figure 1}

(a)
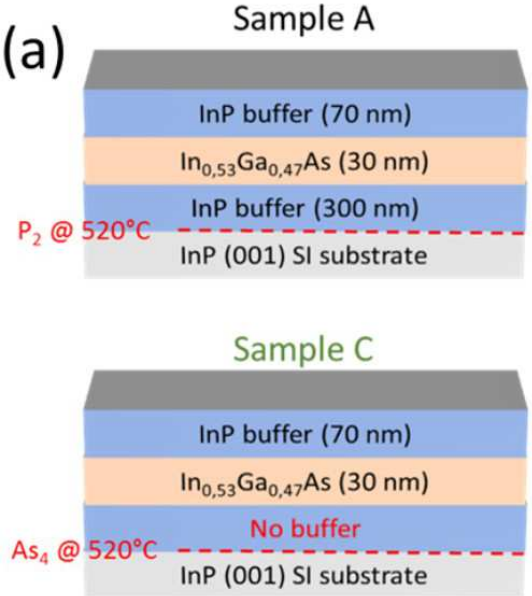

Sample B
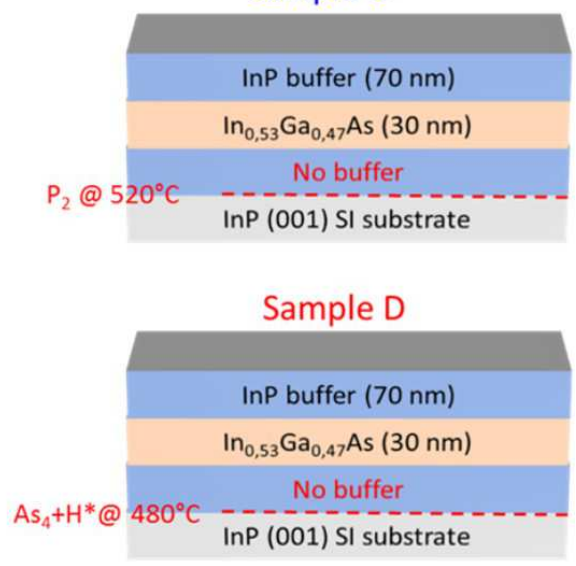

(b)

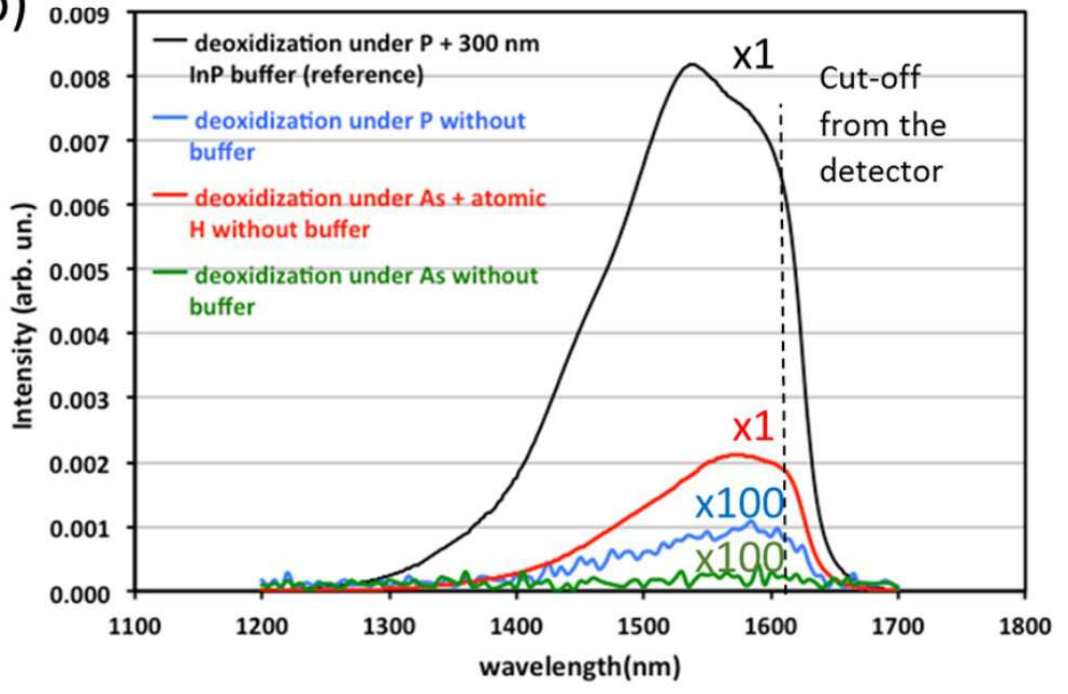


Figure 2

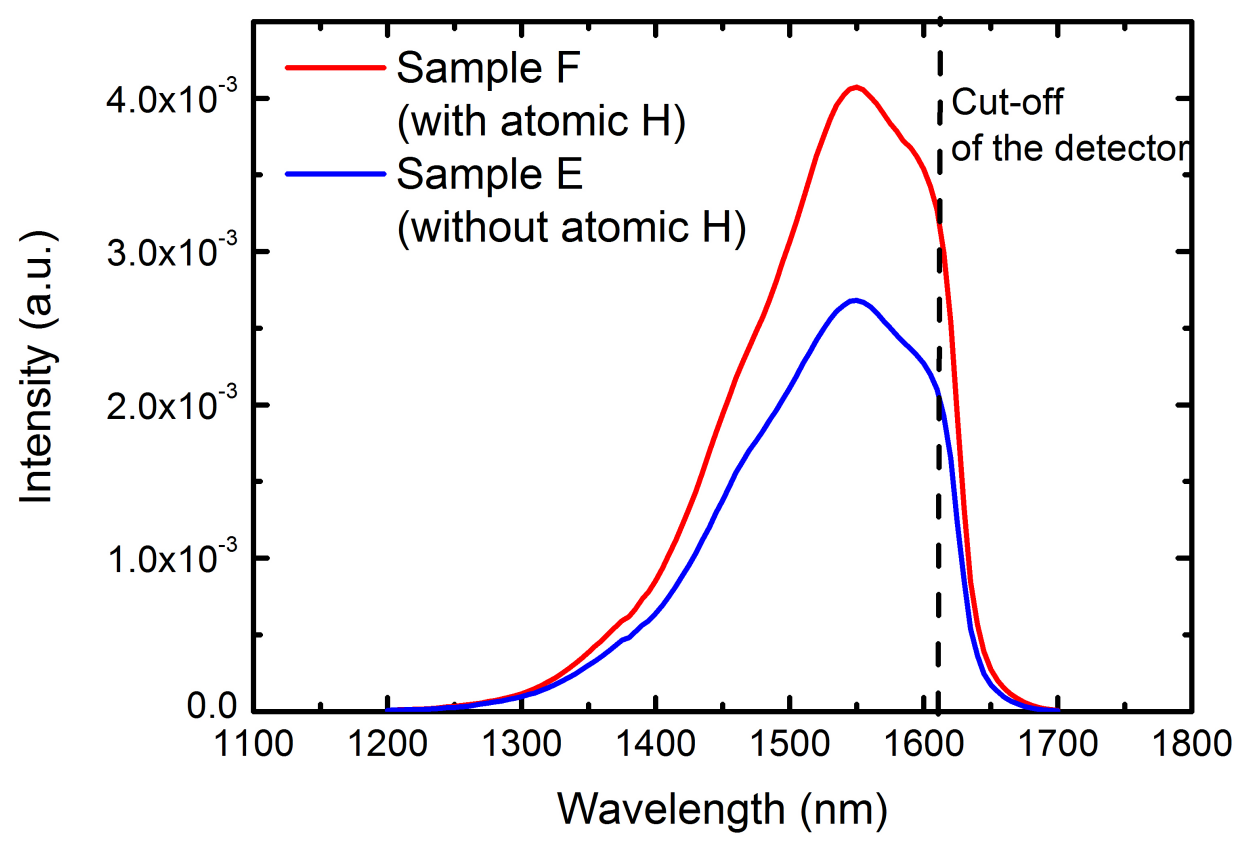


Figure 3

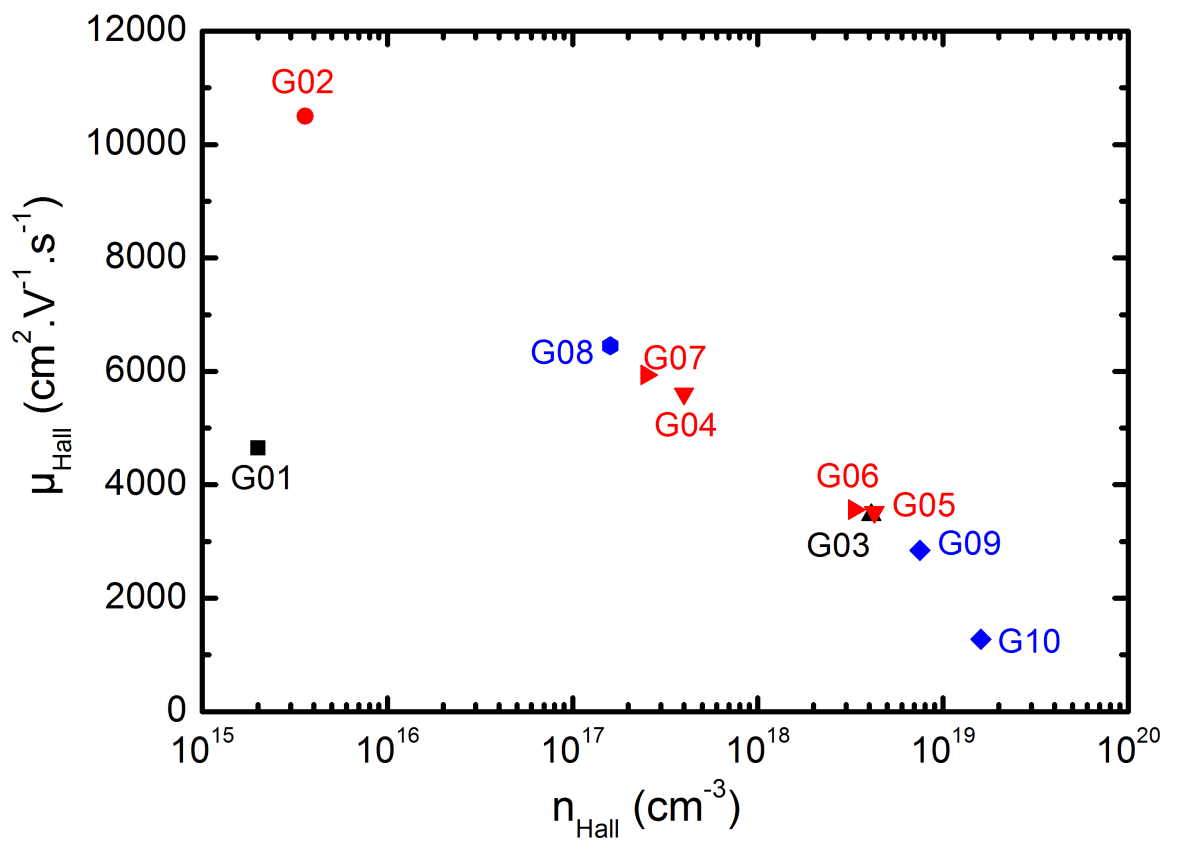


Figure 4

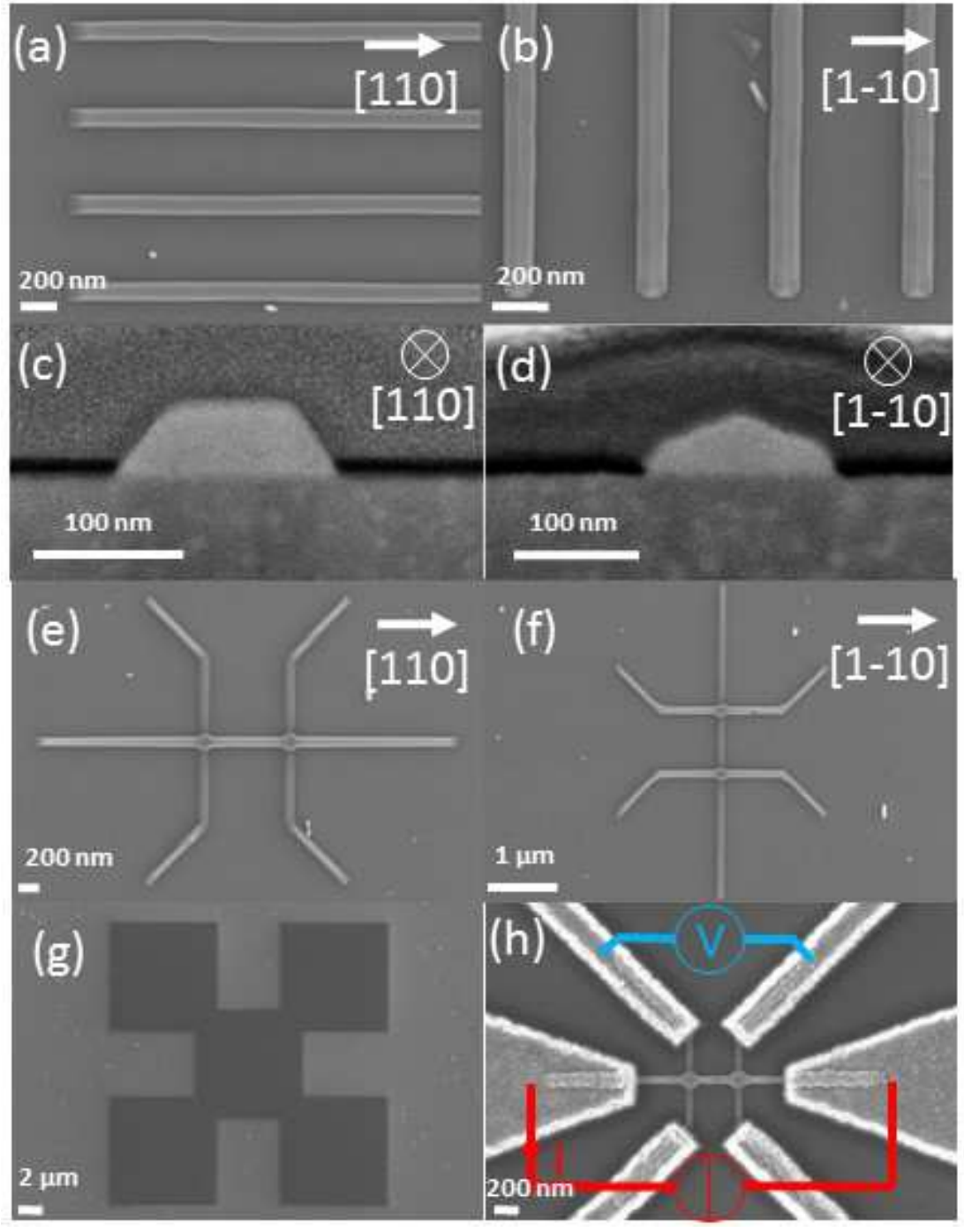


Figure 5

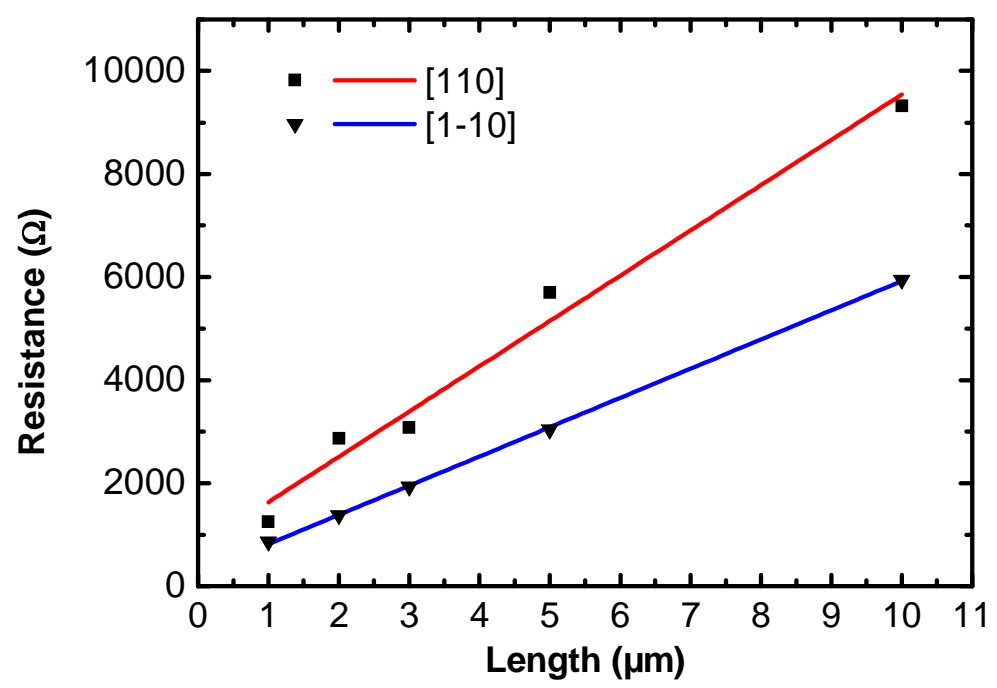


Figure 6

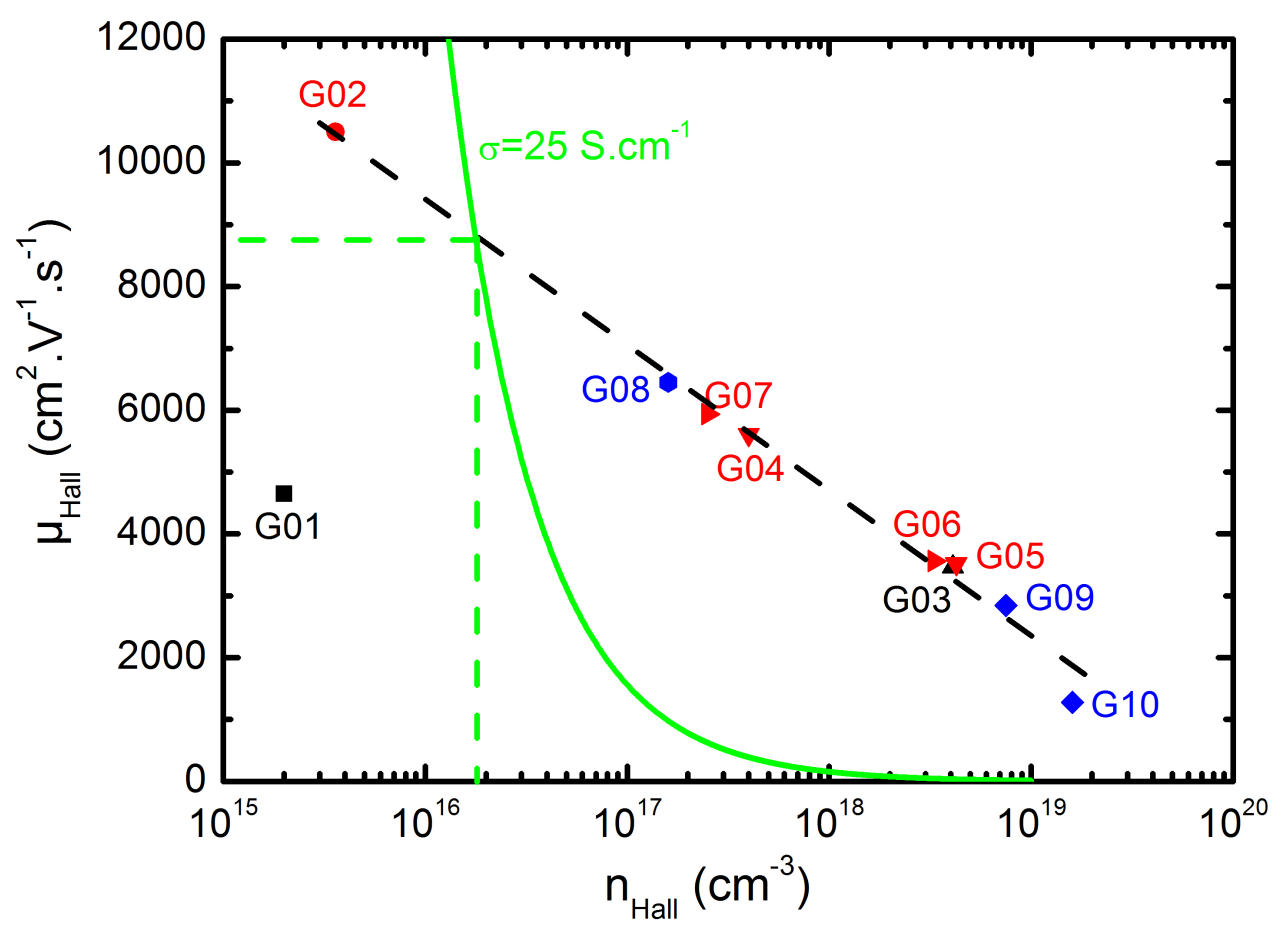

